\title{
Model Systems for Flavoenzyme Activity: Relationships between cofactor structure, binding and redox properties.
}

Yves-Marie Legrand, ${ }^{1,2}$ Mark Gray, ${ }^{1}$ Graeme Cooke ${ }^{2, *}$ and Vincent M. Rotello ${ }^{1, *}$

Contribution from ${ }^{1}$ Department of Chemistry, University of Massachusetts, Amherst, MA 01003, and ${ }^{2}$ Centre for Biomimetic Design and Synthesis, Chemistry,William H. Perkin Building, School of Engineering \& Physical Sciences, Heriot-Watt University, Riccarton, Edinburgh, UK EH14 4AS.

\section{Supplementary Material}

\section{Flavin Synthesis}

Many routes have been developed to produce the flavin ring system, several of which were utilized according to starting material availability to produce the ten artificial cofactors used in this study. These flavins were chosen as they span a wide range of both electron donating and electron withdrawing functionality at the $\mathrm{C} 7$ and $\mathrm{C} 8$ positions. The halogen containing flavins 1a-c were synthesized starting from nitration of the halobenzenes $\mathbf{2 a - c}$ (Scheme 1). The products were then subjected to nucleophilic aromatic substitution at the halogen ortho to the nitro group yielding nitroanilines $\mathbf{4 a - c}$. The nitro group was then reduced to the corresponding aniline (5a-c) using stannous chloride. Reaction of these materials with alloxan 6 under acidic conditions gave the haloflavins 1a-c. Further nucleophilic substitution reactions of these flavins allowed the synthesis of flavins $\mathbf{1 f}, \mathbf{1 g}$ and $\mathbf{1} \mathbf{j}$ to be achieved.

The route chosen for flavins $\mathbf{1 d}, \mathbf{1 e}$ and $\mathbf{1 h}$, containing only protons or methyl groups at the positions under examination started from the appropriate 2-nitroanilines 7a-c. Acylation of these anilines with isobutyryl chloride in the presence of triethylamine afforded the nitro-amides 8a-c, and subsequently the monoacylated dianilines 9a-c were isolated following catalytic hydrogenation. Further reduction of the amide group using LAH gave the alkylamines 5d-f, which formed the desired flavins after reaction with alloxan under acidic conditions.

Finally, production of the 7,8-dimethoxyflavin 1i required a separate synthetic route (Scheme 2). This route began with the reaction of the dimethoxyaniline $\mathbf{1 0}$ with an alkyl halide in the presence of base. Further reaction with chlorouracil 12 afforded compound 13. This material gave the N-oxide 14 after reaction with sodium nitrite in the presence of acid. The desired flavin was obtained following reaction with sodium thiosulfite. 


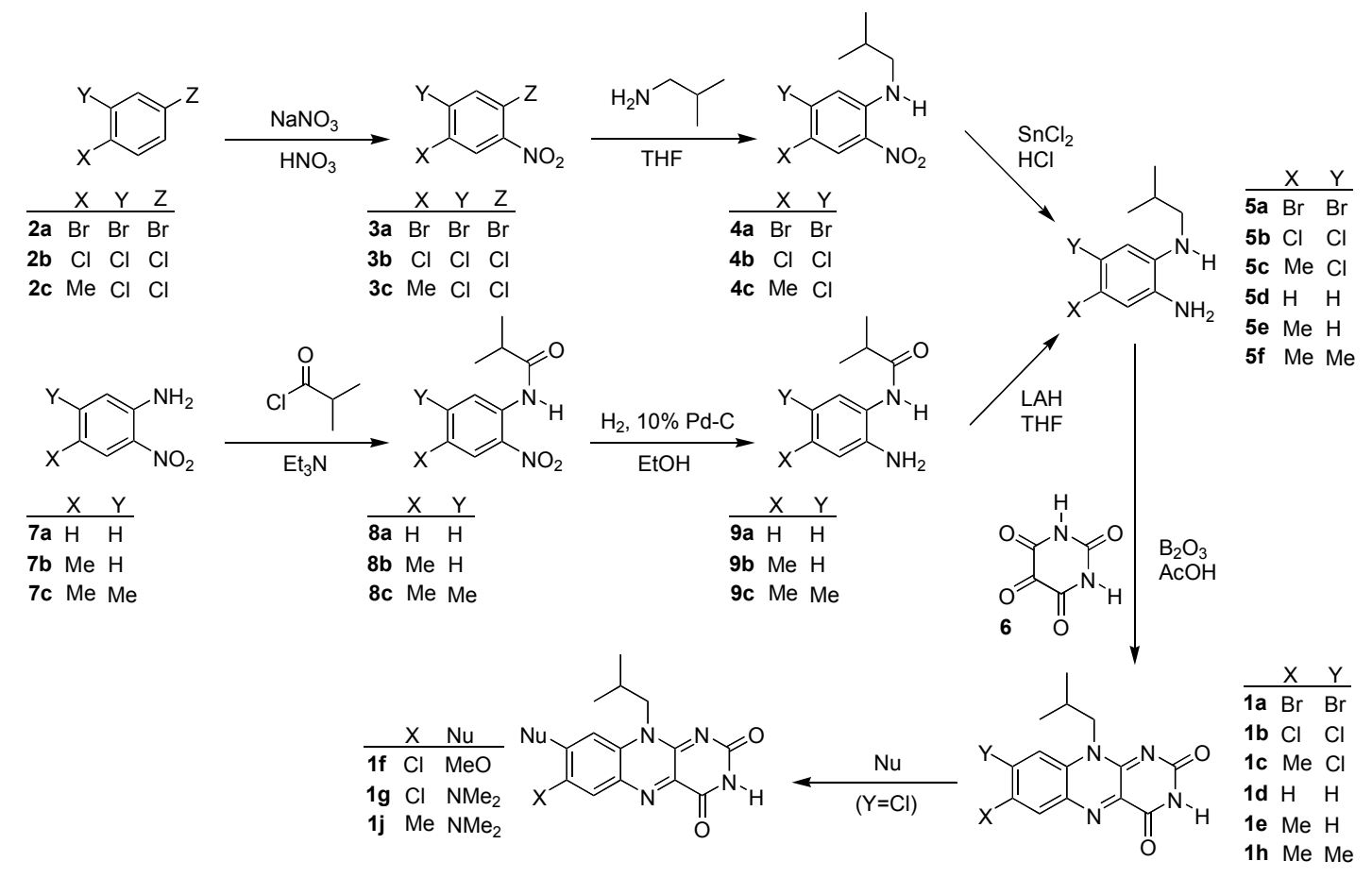

Scheme 1. Synthesis of flavins $\mathbf{1 a - h}$ and $\mathbf{1 j}$
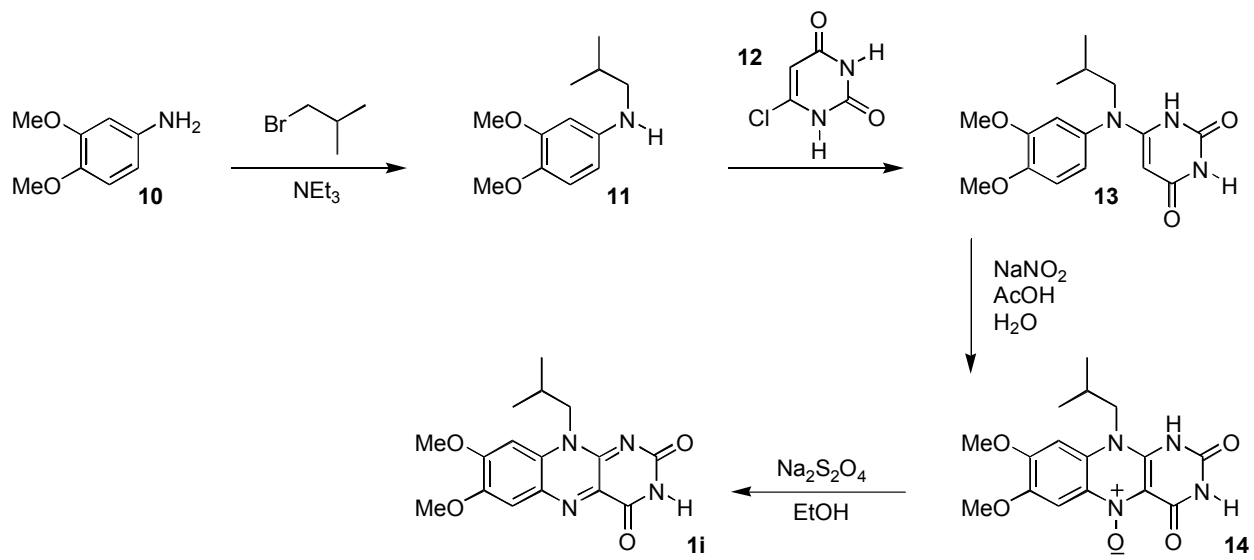

Scheme 2. Synthesis of flavin 1i.

\section{Synthesis}

7,8-dibromo-N(10)-isobutylisoalloxazine (1a). 5a (0.9 g, $3 \mathrm{mmol})$ Was dissolved in glacial acetic acid $(10 \mathrm{~mL})$. Alloxan $(0.5 \mathrm{~g}, 3 \mathrm{mmol})$ and boron oxide $(0.4 \mathrm{~g}, 6 \mathrm{mmol})$ were added. After flushing the system with argon, the reaction mixture was stirred at $60{ }^{\circ} \mathrm{C}$ for 3 hours. Water $(50 \mathrm{~mL})$ was added, and a small amount of particulate matter was re-dissolved via heating. On cooling, a yellow /green precipitate formed. 
The solid was collected by vacuum filtration then further purified using flash column chromatography (silica gel, eluent: $10 \%$ tetrahydrofuran in methylene chloride). The solvent was concentrated and the solid recrystallized twice from absolute ethanol to yield the bright yellow product $(0.3 \mathrm{~g}, 0.7 \mathrm{mmol}, 22 \%)$ : decomposed at $310{ }^{\circ} \mathrm{C}$ without melting; ${ }^{1} \mathrm{H}$ NMR (200 MHz, $\left.\mathrm{CDCl}_{3}\right) \square 8.54(1 \mathrm{H}, \mathrm{s}), 8.53$ (1H, s), 7.91 $(1 \mathrm{H}, \mathrm{s}), 4.54(2 \mathrm{H}, \mathrm{d}), 2.41(1 \mathrm{H}, \mathrm{m}), 1.06(6 \mathrm{H}, \mathrm{d}) ; \mathrm{MS}(\mathrm{EI}): \mathrm{m} / \mathrm{z} 428\left(\mathrm{M}^{+}\right) ;{ }^{13} \mathrm{C}$ NMR (d-DMSO, ppm): $20.516,27.482,51.277,121.266,122.268,131.384,133.827,135.422,135.988,141.008,151.924$, 156.342, 160.269; analysis calculated for $\mathrm{C}_{14} \mathrm{H}_{12} \mathrm{~N}_{4} \mathrm{O}_{2} \mathrm{Br}_{2}$ : C 39.25\%, H 2.80\%, N 13.08\%. Found C $39.18 \%, \mathrm{H} 2.79 \%, \mathrm{~N} 12.79 \%$.

7,8-dichloro-N(10)-isobutylisoalloxazine (1b). Was synthesized using the method described for 1 a. Starting material $\mathbf{5 b}(2.5 \mathrm{~g}, 11 \mathrm{mmol})$. The product was isolated as a bright yellow solid $(2.3 \mathrm{~g}, 7 \mathrm{mmol}$, 63\%); mp 299-300 ${ }^{\circ} \mathrm{C} ;{ }^{1} \mathrm{H}$ NMR $\left(200 \mathrm{MHz}, \mathrm{CDCl}_{3}\right) \square 8.65(1 \mathrm{H}, \mathrm{s}), 8.39(1 \mathrm{H}, \mathrm{s}), 7.73(1 \mathrm{H}, \mathrm{s}), 4.56(2 \mathrm{H}, \mathrm{d})$, 2.42 (1H, m), 1.07 (6H, d); MS(EI): m/z $339\left(\mathrm{M}^{+}\right) ;{ }^{13} \mathrm{C}$ NMR (d-DMSO, ppm): $\square 20.499,27.464,51.409$, 119.337, 129.006, 133.042, 133.605, 134.877, 137.988, 141.059, 151.946, 156.332, 160.246; analysis calculated for $\mathrm{C}_{14} \mathrm{H}_{12} \mathrm{~N}_{4} \mathrm{O}_{2} \mathrm{Cl}_{2}$ : C 49.56\%, H 3.54\%, N 16.52\%. Found C 49.50\%, H 3.63\%, N 16.26\%.

7-methyl-8-chloro-N(10)-isobutylisoalloxazine (1c). Was synthesized using the method described for 1a. Starting material 5c $(0.7 \mathrm{~g}, 3 \mathrm{mmol})$. The product was isolated as a fine yellow powder $(0.7 \mathrm{~g}, 2 \mathrm{mmol}$, $68 \%$ ): decomposed at $320{ }^{\circ} \mathrm{C}$ without melting; ${ }^{1} \mathrm{H}$ NMR $\left(200 \mathrm{MHz}, \mathrm{CDCl}_{3}\right) \square 8.65(1 \mathrm{H}, \mathrm{s}), 8.16(1 \mathrm{H}, \mathrm{s})$, $7.63(1 \mathrm{H}, \mathrm{s}), 4.56(2 \mathrm{H}, \mathrm{d}), 2.54(3 \mathrm{H}, \mathrm{s}), 2.44(1 \mathrm{H}, \mathrm{m}), 1.04(6 \mathrm{H}, \mathrm{d}) ; \mathrm{MS}(\mathrm{EI}): \mathrm{m} / \mathrm{z} \mathrm{318/320}\left(\mathrm{M}^{+}\right) ;{ }^{13} \mathrm{C} \mathrm{NMR}$ (d-DMSO, ppm): $\square 19.921,20.532,27.498,51.256,117.528,132.879,133.808,134.563,134.714$, 139.803, 141.28, 151.734, 156.401, 160.533; analysis calculated for $\mathrm{C}_{15} \mathrm{H}_{12} \mathrm{~N}_{4} \mathrm{O}_{2} \mathrm{Cl}$ : C 56.52\%, $\mathrm{H} 4.74 \%$, N $17.58 \%$. Found C 56.65\%, H 4.83\%, N $17.69 \%$.

N(10)-isobutylisoalloxazine (1d). Was synthesized using the method described for 1a. Starting material 5d (1.2 g, $7 \mathrm{mmol})$. The product was isolated as a bright yellow powder $(0.7 \mathrm{~g}, 3 \mathrm{mmol}, 36 \%)$ : mp $304-$ $306{ }^{\circ} \mathrm{C} ;{ }^{1} \mathrm{H}$ NMR $\left(200 \mathrm{MHz}, \mathrm{CDCl}_{3}\right) \square 8.55(1 \mathrm{H}, \mathrm{s}), 8.35(1 \mathrm{H}, \mathrm{d}), 7.95(1 \mathrm{H}, \mathrm{t}), 7.65(2 \mathrm{H}, \mathrm{t}), 4.77(2 \mathrm{H}, \mathrm{s})$, $2.46(1 \mathrm{H}, \mathrm{m}), 1.04(6 \mathrm{H}, \mathrm{d}) ; \mathrm{MS}(\mathrm{EI}): \mathrm{m} / \mathrm{z} 270.1\left(\mathrm{M}^{+}\right) ;{ }^{13} \mathrm{C}$ NMR (d-DMSO, ppm): $\mathrm{2}$ 20.681, 27.701, 
$51.213,117.752,126.816,132.747,133.778,135.614,135.739,139.649,151.946,156.513,136.649$; analysis calculated for $\mathrm{C}_{14} \mathrm{H}_{14} \mathrm{~N}_{4} \mathrm{O}_{2}$ : C $62.22 \%, \mathrm{H} 5.19 \%, \mathrm{~N} 20.74 \%$. Found $\mathrm{C} 61.88 \%, \mathrm{H} 5.22 \%, \mathrm{~N}$ $20.52 \%$

7-methyl-N(10)-isobutylisoalloxazine (1e). Was synthesized using the method described for 1a. Starting material 5e $(90 \mathrm{mg}, 0.5 \mathrm{mmol})$. The product was isolated as a yellow solid (92 $\mathrm{mg}, 0.8 \mathrm{mmol}, 62 \%)$ : decomposed at $315{ }^{\circ} \mathrm{C}$ without melting; ${ }^{1} \mathrm{H}$ NMR (200 MHz, $\left.\mathrm{CDCl}_{3}\right) \square 8.51(1 \mathrm{H}, \mathrm{s}), 8.11(1 \mathrm{H}, \mathrm{d}), 7.70$ $(1 \mathrm{H}, \mathrm{d}), 7.59(2 \mathrm{H}, \mathrm{d}), 4.61(2 \mathrm{H}, \mathrm{s}), 2.56(3 \mathrm{H}, \mathrm{s}), 2.41(1 \mathrm{H}, \mathrm{m}), 1.03(6 \mathrm{H}, \mathrm{d}) ; \mathrm{MS}(\mathrm{EI}): \mathrm{m} / \mathrm{z} 284\left(\mathrm{M}^{+}\right) ;{ }^{13} \mathrm{C}$ NMR (d-DMSO, ppm): $\square$ 20.624, 21.009, 27.672, 51.246, 117.513, 131.782, 131.842, 135.834, 136.742, $137.147,139.42,151.596,156.44,160.69$; analysis calculated for $\mathrm{C}_{15} \mathrm{H}_{16} \mathrm{~N}_{4} \mathrm{O}_{2}$ : $\mathrm{C} 63.37 \%, \mathrm{H} 5.67 \%, \mathrm{~N}$ 19.71\%. Found C 63.10\%, H 5.65\%, N 19.43\%.

1-nitro-2,4,5-tribromo-benzene (3a). To a solution of sodium nitrate (4 g, $48 \mathrm{mmol}$ ) in concentrated sulfuric acid $(110 \mathrm{~mL})$ was added tribromobenzene $\mathbf{2 a}(10 \mathrm{~g}, 32 \mathrm{mmol})$ over 20 minutes maintaining the reaction temperature below $40{ }^{\circ} \mathrm{C}$. The resulting mixture was stirred at $40{ }^{\circ} \mathrm{C}$ for 3 hours, then cooled to 0 ${ }^{\circ} \mathrm{C}$ before neutralization with sodium hydroxide solution $(2 \mathrm{M})$. The solution was filtered and the collected solid washed with water. The desired material was obtained after recrystallization from petroleum ether as fine pale yellow crystals (8.0 g, $22 \mathrm{mmol}, 69 \%)$ : $\mathrm{mp} 91-93{ }^{\circ} \mathrm{C} ;{ }^{1} \mathrm{H} \mathrm{NMR}\left(200 \mathrm{MHz}, \mathrm{CDCl}_{3}\right) \square 8.13(1 \mathrm{H}, \mathrm{s})$, $8.03(1 \mathrm{H}, \mathrm{s}) ; \mathrm{MS}(\mathrm{EI}): \mathrm{m} / \mathrm{z} 359 / 361\left(\mathrm{M}^{+}\right)$.

1-nitro-2,4,5-trichloro-benzene (3b). Was synthesized using the method described for 3a. Starting material $\mathbf{2 b}(6.9 \mathrm{~mL}, 55 \mathrm{mmol})$. The product was isolated as fine pale yellow crystals $(12 \mathrm{~g}, 52 \mathrm{mmol}$, 94\%): $\mathrm{mp} 55-56{ }^{\circ} \mathrm{C} ;{ }^{1} \mathrm{H}$ NMR (200 MHz, $\left.\mathrm{CDCl}_{3}\right) \square 8.05$ (1H, s), $7.70(1 \mathrm{H}, \mathrm{s}) ; \mathrm{MS}(\mathrm{EI}): \mathrm{m} / \mathrm{z} 225 / 227\left(\mathrm{M}^{+}\right)$. 2,4-dichloro-5-nitrotoluene (3c). Was synthesized using the method described for 3a. Starting material 2c $(10 \mathrm{~g}, 62 \mathrm{mmol})$. The product was isolated as pale yellow crystals $(5.5 \mathrm{~g}, 26 \mathrm{mmol}, 43 \%): \mathrm{mp} 45-46{ }^{\circ} \mathrm{C}$; ${ }^{1} \mathrm{H}$ NMR $\left(200 \mathrm{MHz}, \mathrm{CDCl}_{3}\right) \square 7.80(1 \mathrm{H}, \mathrm{s}), 7.51(1 \mathrm{H}, \mathrm{s}) 2.42(3 \mathrm{H}, \mathrm{s})$.

4,5-dibromo-N-isobutyl-2-nitroaniline (4a). To a solution of 1-nitro-2,4,5-tribromobenzene 3a (1.5 g, 4 $\mathrm{mmol})$ in THF $(100 \mathrm{~mL})$ was added isobutyl amine $(2 \mathrm{~mL}, 21 \mathrm{mmol})$ and triethylamine $(0.6 \mathrm{~mL}, 6 \mathrm{mmol})$. 
The solution was stirred under reflux for 15 hours. The solution was concentrated in vacuo and purified using flash column chromatography (silica gel, eluent: $5 \%$ dichloromethane in petroleum ether) to yield bright orange crystals $(1.2 \mathrm{~g}, 3.41 \mathrm{mmol}, 82 \%)$ : $\mathrm{mp} 81-82{ }^{\circ} \mathrm{C},{ }^{1} \mathrm{H}$ NMR $\left(200 \mathrm{MHz}, \mathrm{CDCl}_{3}\right) \square 8.40(1 \mathrm{H}, \mathrm{s})$, $8.03(1 \mathrm{H}, \mathrm{s}), 7.15(1 \mathrm{H}, \mathrm{s}), 3.09(2 \mathrm{H}, \mathrm{t}), 2.01(1 \mathrm{H}, \mathrm{m}), 1.05(6 \mathrm{H}, \mathrm{d}) ; \mathrm{MS}(\mathrm{EI}): \mathrm{m} / \mathrm{z} 252\left(\mathrm{M}^{+}\right)$.

4,5-dichloro-N-isobutyl-2-nitroaniline (4b). Was synthesized using the method described for $4 \mathbf{a}$. Starting material $\mathbf{3 b}(5 \mathrm{~g}, 22 \mathrm{mmol})$. The product was isolated as orange-red crystals $(4.2 \mathrm{~g}, 16 \mathrm{mmol}$, 72\%): mp 56-57 ${ }^{\circ} \mathrm{C} ;{ }^{1} \mathrm{H}$ NMR $\left(200 \mathrm{MHz}, \mathrm{CDCl}_{3}\right) \square 8.22(1 \mathrm{H}, \mathrm{s}), 8.00(1 \mathrm{H}, \mathrm{s}), 6.90(1 \mathrm{H}, \mathrm{s}), 3.02(2 \mathrm{H}, \mathrm{t})$, $1.95(1 \mathrm{H}, \mathrm{m}), 1.00(6 \mathrm{H}, \mathrm{d}) ; \mathrm{MS}(\mathrm{EI}): \mathrm{m} / \mathrm{z} 262 / 264\left(\mathrm{M}^{+}\right)$.

5-chloro-N-isobutyl-4-methyl-2-nitroaniline (4c). Was synthesized using the method described for $4 \mathbf{a}$. Starting material 3c (1 g, $5 \mathrm{mmol})$. The product was isolated as red-orange crystals $(0.6 \mathrm{~g}, 2.3 \mathrm{mmol}, 50 \%)$ : mp 56-57 ${ }^{\circ} \mathrm{C} ;{ }^{1} \mathrm{H}$ NMR $\left(200 \mathrm{MHz}, \mathrm{CDCl}_{3}\right) \square 8.02(1 \mathrm{H}, \mathrm{s}), 8.00(1 \mathrm{H}, \mathrm{s}), 6.84(1 \mathrm{H}, \mathrm{s}), 3.04(2 \mathrm{H}, \mathrm{t}), 2.27(3 \mathrm{H}$, s), $1.97(1 \mathrm{H}, \mathrm{m}), 1.03(6 \mathrm{H}, \mathrm{d})$.

2-amino-4,5-dibromo-N(1)-isobutylaniline (5a). Zinc powder (5 g, $76 \mathrm{mmol})$ was added in small portions to a suspension of $4 \mathbf{a}(5.3 \mathrm{~g}, 15 \mathrm{mmol})$ in $30 \%$ ammonia $(20 \mathrm{~mL})$ and ethanol $(15 \mathrm{~mL})$ at $0{ }^{\circ} \mathrm{C}$. The resulting mixture was allowed to warm slowly to room temperature and stirred for 5 hours. The zinc was removed by filtration and the remaining solution extracted with ether $(3 \mathrm{x} 40 \mathrm{~mL})$. The organic portions were combined and the solvent removed in vacuo to give an oily residue. This was purified by flash column chromatography (silica gel, eluent: $2 \%$ methylene chloride in petroleum ether) to yield a colorless oil (4.5 g, 14 mmol, 92\%): ${ }^{1} \mathrm{H}$ NMR (200 MHz, $\left.\mathrm{CDCl}_{3}\right) \square 6.92(1 \mathrm{H}, \mathrm{s}), 6.78(1 \mathrm{H}, \mathrm{s}), 3.32(2 \mathrm{H}, \mathrm{s})$, $3.25(1 \mathrm{H}, \mathrm{d}), 2.86(2 \mathrm{H}, \mathrm{t}), 1.90(1 \mathrm{H}, \mathrm{m}), 1.01(6 \mathrm{H}, \mathrm{d}) ; \mathrm{MS}(\mathrm{EI}): \mathrm{m} / \mathrm{z} 322\left(\mathrm{M}^{+}\right)$.

2-amino-4,5-dichloro-N(1)-isobutylaniline (5b). Was synthesized using the method described for $5 \mathbf{a}$. Starting material 4b (4 g, $15 \mathrm{mmol})$. The product was isolated as a colorless oil $(2.7 \mathrm{~g}, 12 \mathrm{mmol}, 77 \%):{ }^{1} \mathrm{H}$ $\operatorname{NMR}\left(200 \mathrm{MHz}, \mathrm{CDCl}_{3}\right) \square 6.75(1 \mathrm{H}, \mathrm{s}), 6.62(1 \mathrm{H}, \mathrm{s}), 3.35(2+1 \mathrm{H}, \mathrm{s}), 2.87(2 \mathrm{H}, \mathrm{t}), 1.90(1 \mathrm{H}, \mathrm{m}), 1.02(6 \mathrm{H}$, d); $\mathrm{MS}(\mathrm{EI}): \mathrm{m} / \mathrm{z} 232 / 234\left(\mathrm{M}^{+}\right)$.

2-amino-5-chloro-N(1)-isobutyl-4-methylaniline (5c). Was synthesized using the method described for 
5a. Starting material $4 \mathbf{c}(1 \mathrm{~g}, 4 \mathrm{mmol})$. The product was isolated as a colorless oil $(0.8 \mathrm{~g}, 3.6 \mathrm{mmol}, 87 \%)$ :

${ }^{1} \mathrm{H}$ NMR $\left(200 \mathrm{MHz}, \mathrm{CDCl}_{3}\right) \square 6.59(1 \mathrm{H}, \mathrm{s}), 6.51(1 \mathrm{H}, \mathrm{s}), 3.25(2+1 \mathrm{H}, \mathrm{s}), 2.82(2 \mathrm{H}, \mathrm{d}), 2.20(3 \mathrm{H}, \mathrm{s}), 1.85$ $(1 \mathrm{H}, \mathrm{m}), 1.00(6 \mathrm{H}, \mathrm{d})$.

2-amino-N-isobutyl-aniline (5d). To a suspension of $\mathrm{LiAlH}_{4}(0.5 \mathrm{~g}, 13 \mathrm{mmol})$ in dry cold tetrahydrofuran $(50 \mathrm{~mL})$ was added $9 \mathrm{a}(0.6 \mathrm{~g}, 3.3 \mathrm{mmol})$ and the reaction was stirred overnight at room temperature. When the reaction was completed the flask was placed in an ice bath. Very slowly, over the course of $15 \mathrm{~min}$, water $(0.5 \mathrm{~mL})$ was added (CAUTION), then $15 \% \mathrm{NaOH}(0.5 \mathrm{~mL})$ and finally another $1.5 \mathrm{~mL}$ of water. A white precipitate was formed, which was filtered and washed 3 times with ether. The filtrate was dried over $\mathrm{MgSO}_{4}$, and the solvent evaporated. The product was isolated as a clear oil $(0.4 \mathrm{~g}$, $2.4 \mathrm{mmol}, 72 \%):{ }^{1} \mathrm{H}$ NMR $\left(200 \mathrm{MHz}, \mathrm{CDCl}_{3}\right) \square 6.89(1 \mathrm{H}, \mathrm{m}), 6.69(3 \mathrm{H}, \mathrm{m}), 3.34(2+1 \mathrm{H}, \mathrm{s}), 2.92(2 \mathrm{H}, \mathrm{d})$, $1.94(1 \mathrm{H}, \mathrm{m}), 1.07(6 \mathrm{H}, \mathrm{d}) ; \mathrm{MS}(\mathrm{EI}): \mathrm{m} / \mathrm{z} 164\left(\mathrm{M}^{+}\right)$.

2-amino-N(1)-isobutyl-4-methyl-aniline (5e). Was synthesized using the method described for $\mathbf{5 d}$. Starting material 9b $(1 \mathrm{~g}, 5 \mathrm{mmol})$. The product was isolated as a colorless oil $(0.78 \mathrm{~g}, 4.4 \mathrm{mmol}, 85 \%):{ }^{1} \mathrm{H}$ NMR $\left(200 \mathrm{MHz}, \mathrm{CDCl}_{3}\right) \square 6.60(3 \mathrm{H}, \mathrm{m}), 3.31(2+1 \mathrm{H}, \mathrm{s}), 2.90(2 \mathrm{H}, \mathrm{d}), 2.25(3 \mathrm{H}, \mathrm{s}), 1.94(1 \mathrm{H}, \mathrm{m}), 1.20$ $(6 \mathrm{H}, \mathrm{d})$.

2-methyl-N-(2-nitrophenyl)-propanamide (8a). 2-Nitroaniline 7a (2.5 g, $18 \mathrm{mmol})$ was dissolved in tetrahydrofuran $(250 \mathrm{~mL})$ under argon. Triethylamine $(3.2 \mathrm{~mL}, 23 \mathrm{mmol})$ and isobutyl chloride $(2.4 \mathrm{~mL}$, $27 \mathrm{mmol}$ ) were slowly added. The reaction mixture was stirred at room temperature for 8 hours and the solvent evaporated. The yellow solid obtained was purified by flash chromatography to afford the pure product (2.82 g, $13.5 \mathrm{mmol}, 75 \%): \mathrm{mp} 70-71{ }^{\circ} \mathrm{C},{ }^{1} \mathrm{H}$ NMR $\left(200 \mathrm{MHz}, \mathrm{CDCl}_{3}\right) \square 10.50(1 \mathrm{H}, \mathrm{s}), 8.85(1 \mathrm{H}$, d), $8.22(1 \mathrm{H}, \mathrm{d}), 7.75(1 \mathrm{H}, \mathrm{t}), 7.18(1 \mathrm{H}, \mathrm{t}), 2.67(1 \mathrm{H}, \mathrm{m}), 1.30(6 \mathrm{H}, \mathrm{d}) ; \mathrm{MS}(\mathrm{EI}) \mathrm{m} / \mathrm{z} 208\left(\mathrm{M}^{+}\right)$.

2-methyl-N-(5-methyl-2-nitrophenyl)-propanamide (8b). Was synthesized using the method described for 8a. Starting material $7 \mathbf{b}(1 \mathrm{~g}, 6.6 \mathrm{mmol})$. The product was isolated as a yellow powder $(1 \mathrm{~g}, 4.9 \mathrm{mmol}$, 74\%): mp 93-94 ${ }^{\circ} \mathrm{C},{ }^{1} \mathrm{H}$ NMR $\left(200 \mathrm{MHz}, \mathrm{CDCl}_{3}\right) \square 10.27(1 \mathrm{H}, \mathrm{s}), 8.58(1 \mathrm{H}, \mathrm{d}), 7.94(1 \mathrm{H}, \mathrm{s}), 7.35(1 \mathrm{H}$, d), $2.57(1 \mathrm{H}, \mathrm{m}), 2.31(3 \mathrm{H}, \mathrm{s}), 1.25(6 \mathrm{H}, \mathrm{d})$. 
N-(2-aminophenyl)-2-methylpropanamide (9a). To a stirred suspension of $\mathbf{8 a}(2.8 \mathrm{~g}, 14 \mathrm{mmol})$ and $10 \% \mathrm{Pd} / \mathrm{C}(0.9 \mathrm{~g})$ in dry ethanol $(30 \mathrm{~mL})$, anhydrous ammonium formate $(4.4 \mathrm{~g}, 70 \mathrm{mmol})$ was added in a single portion. The resulting reaction mixture was stirred at room temperature for $40 \mathrm{~min}$ under argon. The catalyst was removed by filtration and washed with dry ethanol $(10 \mathrm{~mL})$. The filtrate was evaporated under reduced pressure. The resulting residue was triturated with water $(20 \mathrm{~mL})$, the product was extracted with dichloromethane and dried over $\mathrm{NaSO}_{4}$. The organic layer on evaporation gave the desired amino derivative (1.8 g, $10 \mathrm{mmol}, 75 \%): \mathrm{mp} 158-160{ }^{\circ} \mathrm{C} ;{ }^{1} \mathrm{H}$ NMR $\left(200 \mathrm{MHz}, \mathrm{CDCl}_{3}\right) \square 7.30(1 \mathrm{H}, \mathrm{s}), 7.10(2 \mathrm{H}$, m), $6.80(2 \mathrm{H}, \mathrm{m}), 3.85(2 \mathrm{H}, \mathrm{s}), 2.60(1 \mathrm{H}, \mathrm{m}), 1.30(6 \mathrm{H}, \mathrm{d}) ; \mathrm{MS}(\mathrm{EI}) \mathrm{m} / \mathrm{z} 178\left(\mathrm{M}^{+}\right)$.

N-(2-amino-4-methylphenyl)-2-methylpropanamide (9b). Was synthesized using the method described for 9a. Starting material $\mathbf{8 b}(0.5 \mathrm{~g}, 2.3 \mathrm{mmol})$. The product was isolated as a white powder $(0.3 \mathrm{~g}, 1.4$ mmol, 62\%): mp 51-52 ${ }^{\circ} \mathrm{C} ;{ }^{1} \mathrm{H}$ NMR $\left(200 \mathrm{MHz}, \mathrm{CDCl}_{3}\right) \square 7.12(1 \mathrm{H}, \mathrm{s}), 6.90(1 \mathrm{H}, \mathrm{d}), 6.53(2 \mathrm{H}, \mathrm{d}), 3.71$ $(2 \mathrm{H}, \mathrm{s}), 2.48(1 \mathrm{H}, \mathrm{m}), 2.18(3 \mathrm{H}, \mathrm{s}), 1.20(6 \mathrm{H}, \mathrm{d})$.

N-(3,4-dimethoxyphenyl)-2-methylpropanamide (11). 3,4-Dimethoxy-aniline 10 (5 g, 33 mmol) was dissolved in tetrahydrofuran $(200 \mathrm{~mL})$ under argon. Triethylamine $(5.7 \mathrm{~mL}, 41 \mathrm{mmol})$ and then isobutyryl chloride $(5.1 \mathrm{~mL}, 49 \mathrm{mmol})$ was slowly added. The reaction mixture was stirred at room temperature for 8 $\mathrm{h}$ and the solvent evaporated. The yellow solid was purified by flash chromatography (silica gel) to afford pure yellow crystals (6.89 g, $31 \mathrm{mmol}, 95 \%)$ : $\mathrm{mp} 117-118{ }^{\circ} \mathrm{C} ;{ }^{1} \mathrm{H} \mathrm{NMR}\left(200 \mathrm{MHz}, \mathrm{CDCl}_{3}\right) \square 7.46(1 \mathrm{H}, \mathrm{d})$, $7.18(1 \mathrm{H}, \mathrm{s}), 6.81(1 \mathrm{H}, \mathrm{s}), 6.80(1 \mathrm{H}, \mathrm{d}), 3.87(6 \mathrm{H}, \mathrm{d}), 2.49(1 \mathrm{H}, \mathrm{m}), 1.27(6 \mathrm{H}, \mathrm{d}) ; \mathrm{MS}(\mathrm{EI}) \mathrm{m} / \mathrm{z} 223\left(\mathrm{M}^{+}\right)$. 3,4-dimethoxy-N-isobutylaniline (12). To a solution of $\mathrm{LiAlH}_{4}(4.4 \mathrm{~g}, 117 \mathrm{mmol})$ in dry THF $(100 \mathrm{~mL})$ was added $11(6.5 \mathrm{~g}, 29 \mathrm{mmol})$ and stirred overnight at room temperature. When the reaction was completed the flask was place in an ice bath. Very slowly, over the course of $30 \mathrm{~min}$, water $(4.4 \mathrm{~mL})$ was added, then $15 \% \mathrm{NaOH}(4.4 \mathrm{~mL})$ and finally another $13.2 \mathrm{~mL}$ of water. The white precipitate that formed was removed by filtration, and washed with ether $(3 \times 50 \mathrm{~mL})$. The filtrate was dried over $\mathrm{MgSO}_{4}$, and the solvent evaporated. The product was isolated as white flakes (4.75 g, $23 \mathrm{mmol}, 78 \%$ ): $\mathrm{mp} 186-188{ }^{\circ} \mathrm{C} ;{ }^{1} \mathrm{H}$ NMR (200 MHz, CDCl $) \square 6.74(1 \mathrm{H}, \mathrm{d}), 6.25(1 \mathrm{H}, \mathrm{d}), 6.15(1 \mathrm{H}, \mathrm{d}), 3.83(6 \mathrm{H}, \mathrm{d}), 3.46(1 \mathrm{H}, \mathrm{s}), 2.89(2 \mathrm{H}$, 
d), $1.88(1 \mathrm{H}, \mathrm{m}), 0.99(6 \mathrm{H}, \mathrm{d}) ; \mathrm{MS}(\mathrm{EI}) \mathrm{m} / \mathrm{z} 209\left(\mathrm{M}^{+}\right)$.

3,4-dimethoxy-N-isobutyl-N-uracilaniline (13). A mixture of 6-chlorouracil (0.35 g, $2.4 \mathrm{mmol})$ and 12 ( $1 \mathrm{~g}, 4.8 \mathrm{mmol})$ in $5 \mathrm{~mL}$ of dioxane was refluxed for 24 hours. After cooling to room temperature, the solvent was evaporated. The yellow crude powder was recrystallized from ethanol and petroleum ether (1:1) to yield fine colorless crystals (0.6 g, 2mmol, 39\%): mp 187-188 ${ }^{\circ} \mathrm{C} ;{ }^{1} \mathrm{H}$ NMR $\left(200 \mathrm{MHz}, \mathrm{CDCl}_{3}\right) \square$ $10.35(1 \mathrm{H}, \mathrm{s}), 9.94(1 \mathrm{H}, \mathrm{s}), 7.01(1 \mathrm{H}, \mathrm{d}), 6.88(1 \mathrm{H}, \mathrm{s}), 6.80(1 \mathrm{H}, \mathrm{d}), 4.15(1 \mathrm{H}, \mathrm{s}), 3.78(6 \mathrm{H}, \mathrm{d}), 3.49(2 \mathrm{H}$, d), $1.71(1 \mathrm{H}, \mathrm{m}), 0.88(6 \mathrm{H}, \mathrm{d}) ; \mathrm{MS}(\mathrm{EI}) \mathrm{m} / \mathrm{z} 319\left(\mathrm{M}^{+}\right)$.

7,8-dimethoxy-N(10)-isobutylisoalloxazine-N(5)-oxide (14). To a solution of 13 (0.6 g, 2 mmol) in acetic acid $(10 \mathrm{~mL})$ was added in portions $\mathrm{NaNO}_{2}(0.4 \mathrm{~g}, 6 \mathrm{mmol})$ with cooling on ice, providing an orange suspension. After stirring at room temperature for 1.5 hours, the reaction mixture was filtered and washed with ethanol. A recrystallization in chloroform yielded fine orange crystals $(0.63 \mathrm{~g}, 2 \mathrm{mmol}, 96 \%)$ : mp $285{ }^{\circ} \mathrm{C} ;{ }^{1} \mathrm{H}$ NMR $\left(200 \mathrm{MHz}, \mathrm{CDCl}_{3}\right) \square 10.96(1 \mathrm{H}, \mathrm{s}), 7.70(1 \mathrm{H}, \mathrm{s}), 7.24(1 \mathrm{H}, \mathrm{s}), 4.56(2 \mathrm{H}, \mathrm{s}), 3.99(6 \mathrm{H}$, d), $2.34(1 \mathrm{H}, \mathrm{m}), 0.98(6 \mathrm{H}, \mathrm{d})$.

7,8-dimethoxy-N(10)-isobutylisoalloxazine (1i). To a suspension of 14 (0.63 g, $2 \mathrm{mmol})$ in $\mathrm{H}_{2} \mathrm{O} / \mathrm{EtOH}$ $(1: 1,30 \mathrm{~mL})$ was added $\mathrm{Na}_{2} \mathrm{~S}_{2} \mathrm{O}_{4}(0.63 \mathrm{~g}, 3.6 \mathrm{mmol})$. The mixture was stirred open to atmosphere until the initial orange color changed to yellow (5 hours). The crude product was filtered and washed with water. Recrystallization from chloroform yielded bright orange needles (450 mg, 1.4 mmol, 75\%): mp 293-294 ${ }^{\circ} \mathrm{C} ;{ }^{1} \mathrm{H}$ NMR $\left(200 \mathrm{MHz}, \mathrm{CDCl}_{3}\right) \square 8.47(1 \mathrm{H}, \mathrm{s}), 7.66(1 \mathrm{H}, \mathrm{s}), 6.93(1 \mathrm{H}, \mathrm{s}), 4.66(2 \mathrm{H}, \mathrm{s}), 4.07$ (6H, d), 2.47 $(1 \mathrm{H}, \mathrm{m}), 1.07$ (6H, d); MS(EI): m/z $330\left(\mathrm{M}^{+}\right) ;{ }^{13} \mathrm{C}$ NMR (d-DMSO, ppm): $\square 20.802,27.627,51.537$, $57.135,57.858,98.617,111.783,131.036,132.036,135.244,149.462,151.289,156.403,157.17,161.167$; analysis calculated for $\mathrm{C}_{16} \mathrm{H}_{18} \mathrm{~N}_{4} \mathrm{O}_{4}$ : C 58.18\%, H 5.49\%, N 16.97\%. Found C 58.20\%, H 5.73\%, N $16.91 \%$.

7-chloro-8-methoxy-N(10)-isobutylisoalloxazine (1f). To a solution of $\mathbf{1 b}(50 \mathrm{mg}, 0.2 \mathrm{mmol})$ in methanol $(20 \mathrm{~mL})$ was added sodium methoxide in methanol $(30 \%, 0.33 \mathrm{~mL})$. The reaction mixture was heated to $50{ }^{\circ} \mathrm{C}$ and reacted overnight. The solvent was evaporated, and the crude product was partitioned 
between methylene chloride $(100 \mathrm{~mL})$ and water $(100 \mathrm{~mL})$. The methylene chloride was separated, concentrated and the product was crashed out of solution by addition of hexane. The product was isolated as fine yellow needles (35 mg, $0.1 \mathrm{mmol}, 70 \%)$ : decomposed $320{ }^{\circ} \mathrm{C} ;{ }^{1} \mathrm{H}$ NMR $\left(200 \mathrm{MHz}, \mathrm{CDCl}_{3}\right) \square 8.37$ $(1 \mathrm{H}, \mathrm{s}), 8.32(1 \mathrm{H}, \mathrm{s}), 6.95(1 \mathrm{H}, \mathrm{s}), 4.64(2 \mathrm{H}, \mathrm{s}), 4.15(3 \mathrm{H}, \mathrm{s}), 2.47(1 \mathrm{H}, \mathrm{m}), 1.07(6 \mathrm{H}, \mathrm{d}) ; \mathrm{MS}(\mathrm{EI}): \mathrm{m} / \mathrm{z} 335$ $\left(\mathrm{M}^{+}\right) ;{ }^{13} \mathrm{C}$ NMR (d-DMSO, ppm): $\square 20.889,27.55,51.524,58.638,99.829,121.998,131.094,132.945$, 135.238, 137.095, 152.147, 156.494, 160.174, 160.836; analysis calculated for $\mathrm{C}_{15} \mathrm{H}_{15} \mathrm{~N}_{4} \mathrm{O}_{3} \mathrm{Cl}$ : C 53.81\%, H 4.48\%, N 16.74\%. Found C 53.65\%, H 4.56\%, N 16.52\%.

7-methyl-8-dimethylamine-N(10)-isobutylisoalloxazine (1j). To a solution of $\mathbf{1 c}(20 \mathrm{mg}, 0.1 \mathrm{mmol})$ in dimethylformamide $(2 \mathrm{~mL})$ was added dimethylamine $(2 \mathrm{M}, 0.2 \mathrm{~mL})$ in tetrahydrofuran. The reaction mixture was placed in a sealed tube, heated at $100{ }^{\circ} \mathrm{C}$ for 12 hours. Water was added $(150 \mathrm{~mL})$ and the crude product was extracted from the aqueous layer with methylene chloride $(150 \mathrm{~mL})$. The methylene chloride was separated and concentrated to approximately $2 \mathrm{~mL}$. A large excess of distilled hexane (50 $\mathrm{mL}$ ) was added to afford fine red needles $(20 \mathrm{mg}, 0.1 \mathrm{mmol}, 97 \%)$ : decomposed around $285{ }^{\circ} \mathrm{C}$ without melting; ${ }^{1} \mathrm{H}$ NMR $\left(200 \mathrm{MHz}, \mathrm{CDCl}_{3}\right) \square 8.35(1 \mathrm{H}, \mathrm{s}), 7.93(1 \mathrm{H}, \mathrm{s}), 6.71(1 \mathrm{H}, \mathrm{s}), 4.58(2 \mathrm{H}, \mathrm{d}), 3.10(6 \mathrm{H}, \mathrm{s})$, $2.48(3 \mathrm{H}, \mathrm{s}), 2.46(1 \mathrm{H}, \mathrm{m}), 1.03(6 \mathrm{H}, \mathrm{d}) ; \mathrm{MS}(\mathrm{EI}): \mathrm{m} / \mathrm{z} 327\left(\mathrm{M}^{+}\right) ;{ }^{13} \mathrm{C}$ NMR (d-DMSO, ppm): $\square$ 20.809, $20.951,27.434,43.85,50.949,101.919,130.244,131.814,133,134.119,135.192,151.911,156.569$, 159.651, 161.391; analysis calculated for $\mathrm{C}_{17} \mathrm{H}_{21} \mathrm{~N}_{5} \mathrm{O}_{2}$ : C 62.37\%, H 6.47\%, N 21.39\%. Found C 62.38\%, H $6.57 \%$, N $21.51 \%$.

7-chloro-8-dimethylamine-N(10)-isobutylisoalloxazine (1g). Was synthesized using the method described for $\mathbf{1 j}$. Starting material $\mathbf{1 b}(20 \mathrm{mg}, 0.1 \mathrm{mmol})$. The product was isolated as red crystals $(18 \mathrm{mg}$, $0.1 \mathrm{mmol}, 87 \%)$ : decomposed around $310{ }^{\circ} \mathrm{C}$ without melting, ${ }^{1} \mathrm{H} \mathrm{NMR}\left(200 \mathrm{MHz}, \mathrm{CDCl}_{3}\right) \square 8.38(1 \mathrm{H}, \mathrm{s})$, $8.18(1 \mathrm{H}, \mathrm{s}), 6.77(1 \mathrm{H}, \mathrm{s}), 4.55(2 \mathrm{H}, \mathrm{d}), 3.20(6 \mathrm{H}, \mathrm{s}), 2.43(1 \mathrm{H}, \mathrm{m}), 1.05(6 \mathrm{H}, \mathrm{d}) ; \mathrm{MS}(\mathrm{EI}): \mathrm{m} / \mathrm{z}$ 347/349 $\left(\mathrm{M}^{+}\right) ;{ }^{13} \mathrm{C}$ NMR (d-DMSO, ppm): $\square 21.008,27.535,43.937,51.212,104.64,124.481,131.47,134.239$, 134.56, 135.268, 152.233, 155.574, 156.552, 161.03; analysis calculated for $\mathrm{C}_{16} \mathrm{H}_{18} \mathrm{ClN}_{5} \mathrm{O}_{2}: \mathrm{C} 55.25 \%, \mathrm{H}$ $5.22 \%$, N 20.14\%. Found C 54.99\%, H 5.26\%, N $19.92 \%$. 
Cyclic Voltammograms of flavin alone and flavin bound with DAP ( $\mathrm{mV}$ vs Ferrocene)

$-\quad$ free flavin
$-------\quad$ flavin + DAP

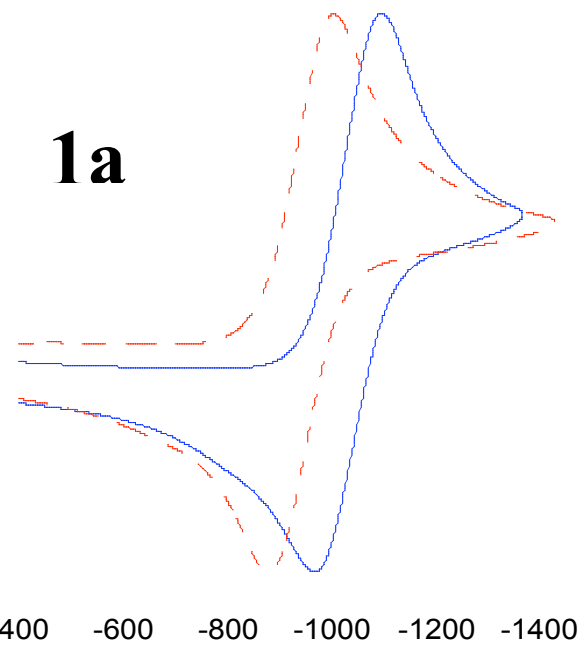

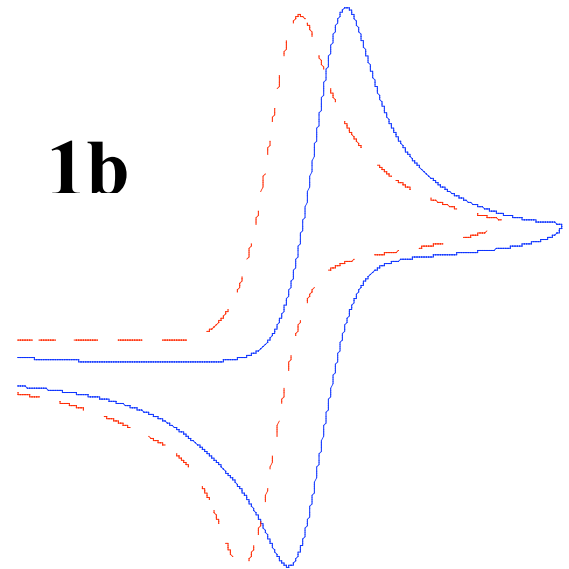

$\begin{array}{llllll}-400 & -600 & -800 & -1000 & -1200 & -1400\end{array}$ 

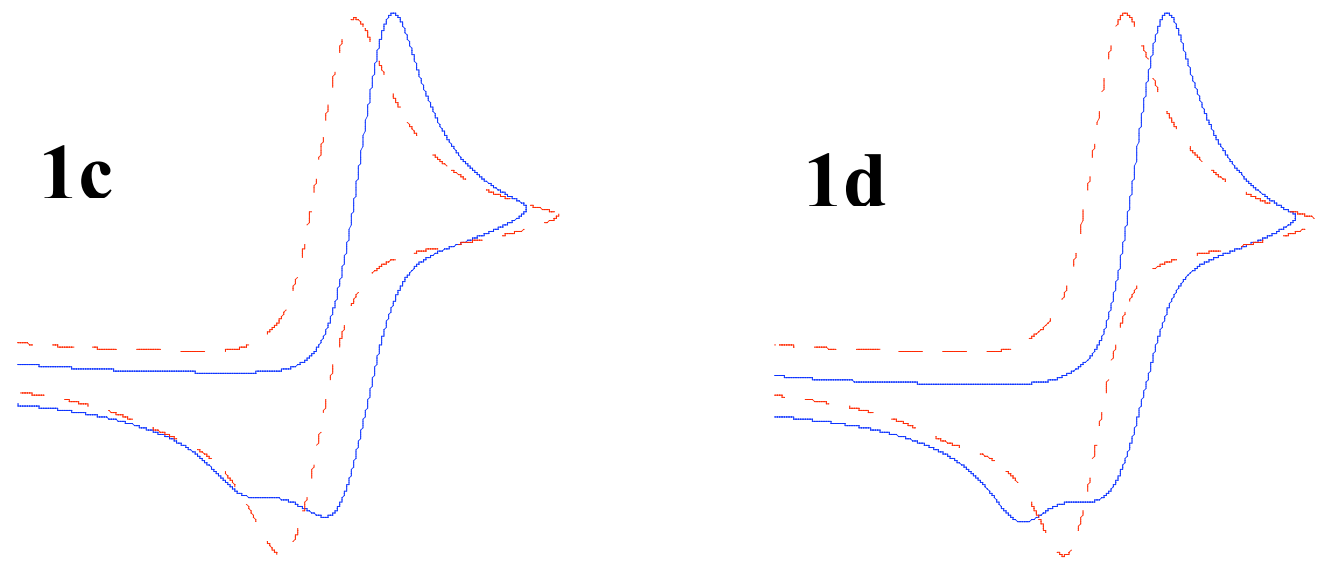

$\begin{array}{llllll}-400 & -600 & -800 & -1000 & -1200 & -1400\end{array}$
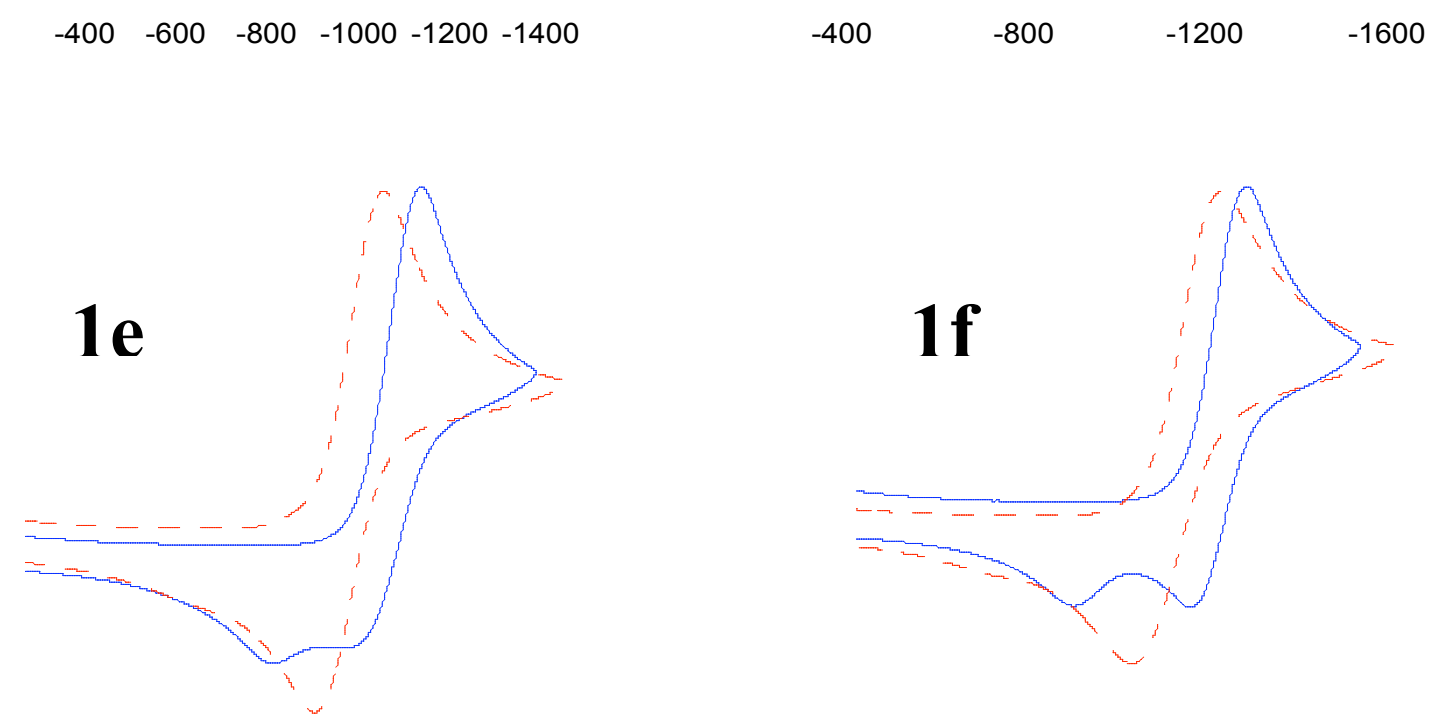

$-400$
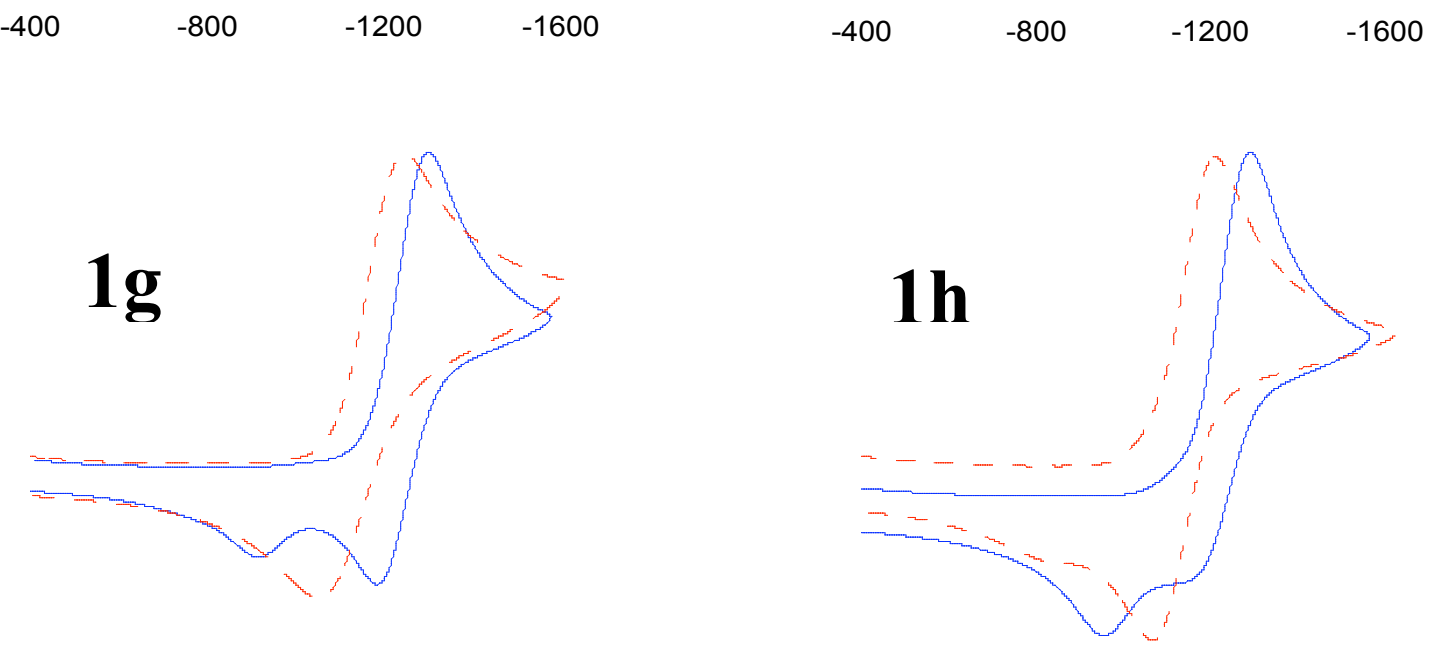

$-400$

$-800$

$-1200$

$-1600$

$-400$

$-800 \quad-1200$

$-1600$ 


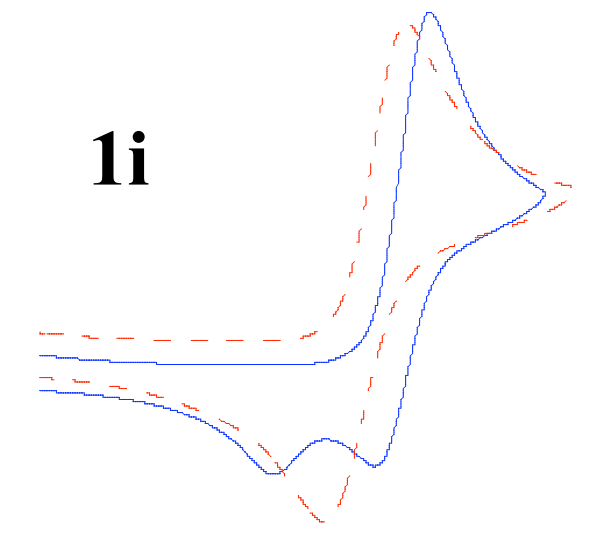

$-400$

$-1200$

$-1600$

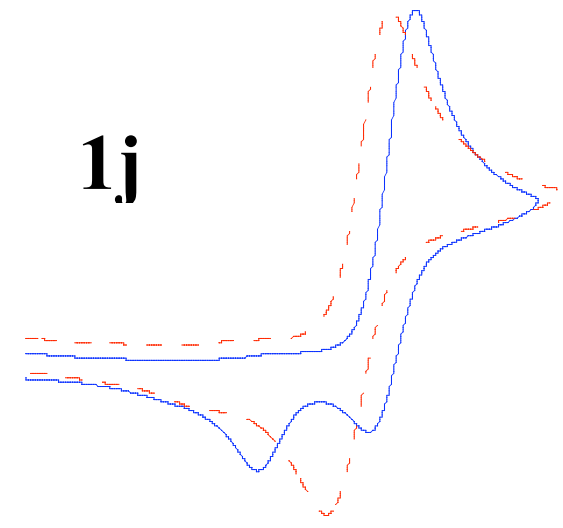

$-400$

$-800$

$-1200$

$-1600$ 